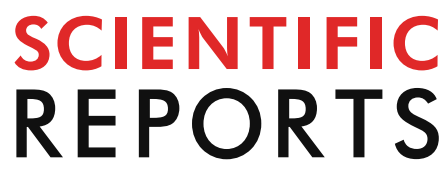

natureresearch

Check for updates

\title{
Risk factors for endothelial cell loss after Descemet membrane endothelial keratoplasty (DMEK)
}

Takahiko Hayashi ${ }^{1,2 凶}$, Silvia Schrittenlocher ${ }^{1}$, Sebastian Siebelmann ${ }^{1}$, Viet Nhat Hung Le ${ }^{1,3}$, Mario Matthaei ${ }^{1}$, Jeremy Franklin ${ }^{4}$, Björn Bachmann ${ }^{1,6}$ \& Claus Cursiefen ${ }^{1,5,6}$

This study aimed to identify the risk factors for endothelial cell density (ECD) loss after Descemet membrane endothelial keratoplasty (DMEK) and analyse whether donor tissues from cold versus organ culture differ in terms of ECD loss after DMEK. Consecutive DMEK cases from a prospective database for Fuchs' endothelial corneal dystrophy were retrospectively analysed between 2011 and 2016 at the University of Cologne, and the possible risk factors for ECD loss, including patient-related factors, type of tamponade (air or $20 \%$ sulphur hexafluoride gas), type of surgery (triple DMEK or DMEK alone), re-bubbling, immune rejection, and donor-related factors were determined. Eight hundred and forty-one eyes were selected. There was no significant difference in the best-corrected visual acuity (logarithm of the minimal angle of resolution) and corneal thickness ( $P=0.540$ and $P=0.667)$ between groups. Immune reactions were more common in cold cultures $(P=0.019)$, but $E C D$ loss (1 year after DMEK) was greater in organ cultures $(38.3 \pm 0.8 \%)$ than in cold cultures $(34.7 \pm 1.4 \%)$ $(P=0.022)$. Only re-bubbling was significantly associated with $E C D$ loss $(P<0.001)$. Re-bubbling was found to be a key factor for ECD loss at 1 year after DMEK.

Descemet membrane endothelial keratoplasty (DMEK) is a widely applied technique for performing keratoplasty and restoring vision in patients with endothelial diseases, such as Fuchs' endothelial corneal dystrophy (FECD) or bullous keratopathy ${ }^{1-5}$. As this technique replaces only the endothelial layer that is attached to the Descemet membrane (DM), surgeons can achieve rapid improvements in visual acuity, better final visual outcomes, and less immunological reactions, compared to other techniques ${ }^{6-9}$, such as Descemet stripping automated endothelial keratoplasty (DSAEK) or penetrating keratoplasty (PKP).

Despite the excellent outcomes, there are a steep short-term decrease in the transplanted endothelial cell density (ECD) for up to 6 months and a gradual decrease after 6 months. The average ECD loss rate at 6 months described in the literature ranges from 29 to $48 \%^{10-12}$. Although many researchers have indicated certain factors associated with ECD loss in both DMEK and PKP or in DSAEK, such as graft diameter ${ }^{13,14}$, surgical indication, type of filling gas ${ }^{15}$, rebubbling, or culture medium ${ }^{16-18}$, there has been no consensus on the most important factor associated with the final ECD outcome.

Regarding the relationship between the condition of the culture and the surgical outcomes, it has been reported in a small number of short-term case series that cold cultures may negatively impact the outcomes ${ }^{18}$. Evaluations in larger cohorts are needed. Furthermore, the difference between organ culture and cold culture needs to be investigated.

This study aimed to identify the risk factors for endothelial cell loss after DMEK by evaluating the correlation between surgical outcomes and clinically relevant factors. To the best of our knowledge, this is the first study to evaluate the factors associated with ECD loss at 1 year after DMEK in a large sample.

\footnotetext{
${ }^{1}$ Department of Ophthalmology, University of Cologne, Cologne, Germany. ${ }^{2}$ Department of Ophthalmology, Yokohama Minami Kyosai Hospital, Kanagawa, Japan. ${ }^{3}$ Department of Ophthalmology, Hue College of Medicine and Pharmacy, Hue University, Hue, Vietnam. ${ }^{4}$ Institute of Medical Statistics and Computational Biology, University of Cologne, Cologne, Germany. ${ }^{5}$ Centre for Molecular Medicine Cologne, CMMC, University of Cologne, Cologne, Germany. ${ }^{6}$ These authors jointly supervised this work: Björn Bachmann and Claus Cursiefen. ${ }^{\square}$ email: takamed@gmail.com
} 


\begin{tabular}{|l|l|l|l|c|}
\hline Factors & Total & Organ culture & Cold culture & P-value (organ vs cold) \\
\hline Numbers of eyes & 841 & 644 & 197 & \\
\hline Sex: female $(\%) /$ male (\%) & $487(57.9) / 354(42.1)$ & $368(57.1) / 276(42.8)$ & $119(60.4) / 78(39.6)$ & 0.440 \\
\hline Age (years) $($ mean \pm SD) & $70.2 \pm 9.1$ & $69.8 \pm 0.4$ & $71.8 \pm 0.6$ & 0.008 \\
\hline Donor sex: female (\%)/male (\%) & $286(38.8) / 451(60.2)$ & $212(37.4) / 355(62.6)$ & $74(43.5) / 96(56.5)$ & 0.150 \\
\hline Donor age (years) (mean \pm SD) & $69.0 \pm 10.9$ & $70.0 \pm 0.4$ & $65.9 \pm 0.8$ & $<0.001$ \\
\hline Storage time (days) (mean \pm SD) & $16.9 \pm 6.1$ & $18.7 \pm 0.2$ & $11.4 \pm 0.4$ & $<0.001$ \\
\hline Lens status: PC-IOL (\%) & $367(43.9)$ & $275(43.2)$ & $91(46.7)$ & 0.574 \\
\hline Type of surgery: triple DMEK (\%) & $464(55.2)$ & $358(55.6)$ & $106(53.8)$ & 0.692 \\
\hline Type of tamponade gas: SF6 (\%) & $408(48.6)$ & $298(46.3)$ & $110(55.8)$ & 0.028 \\
\hline
\end{tabular}

Table 1. Characteristics of the patients. $S D$ standard deviation, $P C-I O L$ posterior chamber intra-ocular lens, $D M E K$ Descemet membrane endothelial keratoplasty, triple DMEK DMEK combined with cataract surgery and intra-ocular lens implantation, SF6 sulphur hexafluoride.

\section{Results}

Patient demographics. Table 1 summarises the characteristics of the patients who underwent DMEK in the current study. A total of 841 eyes from 841 patients were included in this study, which consisted of 354 men and 487 women. The recipients' mean age was $70.2 \pm 9.1$ years. All patients were diagnosed with FECD. A total of $464(55.2 \%)$ patients received triple DMEK, whereas $377(44.8 \%)$ patients were treated with DMEK alone. Regarding tamponade gas, air was used in 432 (51.4\%) eyes and 20\% sulphur hexafluoride (SF6) gas in 409 $(48.6 \%)$ eyes.

Clinical course. The best-corrected visual acuity (BCVA) (logarithm of the minimal angle of resolution [ $\log M A R])$ improved from $1.15 \pm 0.72 \log$ MAR $(20 / 200$ Snellen) preoperatively to $0.31 \pm 0.27 \log$ MAR $(20 / 40$ Snellen) at 1 month, $0.19 \pm 0.17 \log$ MAR (20/32 Snellen) at 3 months, $0.14 \pm 0.10 \log$ MAR (20/30 Snellen) at 6 months, and $0.11 \pm 0.11 \log$ MAR $(20 / 25$ Snellen $)$ at 12 months postoperatively. The central corneal thickness (CCT) decreased from $664.5 \pm 122.9 \mu \mathrm{m}$ preoperatively to $564.3 \pm 107.0 \mu \mathrm{m}$ at $1 \mathrm{month}, 533.1 \pm 56.7 \mu \mathrm{m}$ at 3 months, $533.4 \pm 65.1 \mu \mathrm{m}$ at 6 months, and $534.7 \pm 45.2 \mu \mathrm{m}$ at 12 months postoperatively. The donor corneal ECD decreased from $2,717 \pm 218$ cells $/ \mathrm{mm}^{2}$ preoperatively to $1802 \pm 343$ cells $/ \mathrm{mm}^{2}$ at 1 month, $1757 \pm 367$ cells $/ \mathrm{mm}^{2}$ at 3 months, $1,740 \pm 376$ cells $/ \mathrm{mm}^{2}$ at 6 months, and $1694 \pm 404$ cells $/ \mathrm{mm}^{2}$ at 12 months postoperatively. The ECD loss rates were $33.7 \pm 12.9 \%$ at 1 month, $35.0 \pm 13.6 \%$ at 3 months, $36.0 \pm 14.0 \%$ at 6 months, and $37.5 \pm 14.5 \%$ at 12 months postoperatively. Immune rejection episodes were seen in $14(1.66 \%)$ eyes. Rebubbling procedures were performed in 328 (39.0\%) eyes.

Donor characteristics and comparison between organ culture and cold culture. As shown in Table 2, there were 644 (76.6\%) organ culture donors and 197 (23.4\%) cold culture donors. The mean donor age was $69.0 \pm 10.9$ years. The mean donor age in the organ culture group was $70.0 \pm 0.4$ years, which was significantly higher than that in the cold culture group ( $65.9 \pm 0.8$ years; $\mathrm{P}<0.001$; t-test). Regarding donor sex, $62.6 \%$ were men, and $37.4 \%$ were women. The mean storage time was $18.7 \pm 0.2$ days in the organ culture group, as opposed to $11.4 \pm 0.4$ days in the cold culture group. There was a significant difference between the organ and cold culture groups $(\mathrm{P}<0.001$; t-test $)$.

The ECD in the organ culture was 2,734 \pm 9 (baseline ECD; $\mathrm{N}=636$ ), $1766 \pm 20$ ( 1 month, $\mathrm{N}=287$ ), $1752 \pm 23$ (3 months, $\mathrm{N}=246), 1712 \pm 21$ (6 months, $\mathrm{N}=303)$, and $1681 \pm 21(12$ months, $\mathrm{N}=357)$; that in the cold culture was 2,663 \pm 15 (baseline ECD, $\mathrm{N}=197$ ), $1872 \pm 37$ ( 1 month, $\mathrm{N}=84$ ), $1773 \pm 41$ ( 3 months, $\mathrm{N}=78$ ), $1833 \pm 40$ (6 months, $\mathrm{N}=86$ ), and $1741 \pm 39$ (12 months, $\mathrm{N}=108)$. The ECD was significantly higher in the organ culture than in the cold culture at 1 month $(\mathrm{P}=0.012$, $\mathrm{t}$-test $)$ and 6 months postoperatively $(\mathrm{P}=0.008, \mathrm{t}$-test $)$. However, as the baseline ECD was significantly higher in the organ culture $(\mathrm{P}<0.001$, $\mathrm{t}$-test $)$, organ culture resulted in a significantly greater ECD loss $(\mathrm{P}<0.001$ at 1 month, $\mathrm{P}=0.248$ at 3 months, $\mathrm{P}=0.003$ at 6 months, and $\mathrm{P}=0.022$ at 12 months postoperatively; t-test).

As shown in Fig. 1, the ECD loss rates in the organ culture were $33.5 \pm 0.8 \%$ at 1 month $(\mathrm{N}=287), 33.9 \pm 0.9 \%$ at 3 months $(\mathrm{N}=246), 35.6 \pm 0.8 \%$ at 6 months $(\mathrm{N}=302)$, and $36.8 \pm 0.8 \%$ at 12 months $(\mathrm{N}=357)$ postoperatively. Those in the cold culture were $26.6 \pm 1.4 \%$ at 1 month $(\mathrm{N}=84), 30.5 \pm 1.5 \%$ at 3 months $(\mathrm{N}=78), 29.2 \pm 1.5 \%$ at 6 months $(\mathrm{N}=86)$, and $31.9 \pm 1.4 \%$ at 12 months $(\mathrm{N}=108)$ postoperatively.

The prognosis of the BCVA was similar in the two groups (Fig. 2), whereas a significant difference was noted only at 1 month postoperatively $(\mathrm{P}=0.002$, $\mathrm{t}$-test $)$. The transition of the CCT was also similar in the two groups (Fig. 3), whereas the CCT was significantly higher in the cold culture at 1 month postoperatively $(\mathrm{P}=0.036)$. A longer culture time had a negative impact on ECD loss in the cold culture (Fig. 4).

There was no significant difference in re-bubbling (yes/no) between the two groups $(\mathrm{P}=0.165$, Pearson's chi-squared test). The immune rejection rate was significantly higher in the cold culture $(\mathrm{P}=0.019$, Pearson's chi-squared test). The percentage of the use of $20 \%$ SF6 gas was $46.3 \%$ in the organ culture and $55.8 \%$ in the cold culture $(\mathrm{P}<0.001$, Pearson's chi-squared test $)$. 


\begin{tabular}{|c|c|c|c|}
\hline Factors & Organ culture & Cold culture & P-value \\
\hline Baseline ECD $\left(\right.$ cells $\left./ \mathrm{mm}^{2}\right)($ mean \pm SD $)$ & $2,734 \pm 9(\mathrm{n}=636)$ & $2,663 \pm 15(n=197)$ & $<0.001$ \\
\hline ECD 1 month after surgery $\left(\right.$ cells $\left./ \mathrm{mm}^{2}\right)($ mean \pm SD $)$ & $1766 \pm 20(n=287)$ & $1872 \pm 37(\mathrm{n}=84)$ & 0.012 \\
\hline ECD 3 months after surgery $\left(\right.$ cells $\left./ \mathrm{mm}^{2}\right)($ mean \pm SD $)$ & $1752 \pm 23(n=246)$ & $1773 \pm 41(\mathrm{n}=78)$ & 0.661 \\
\hline ECD 6 months after surgery $\left(\right.$ cells $\left./ \mathrm{mm}^{2}\right)($ mean \pm SD $)$ & $1712 \pm 21(\mathrm{n}=303)$ & $1833 \pm 40(\mathrm{n}=86)$ & 0.001 \\
\hline ECD 12 months after surgery $\left(\right.$ cells $\left./ \mathrm{mm}^{2}\right)($ mean \pm SD $)$ & $1681 \pm 21(\mathrm{n}=357)$ & $1741 \pm 39(\mathrm{n}=108)$ & 0.179 \\
\hline ECD loss rates at 12 months (\%) & $38.3 \pm 0.8(\mathrm{n}=357)$ & $34.7 \pm 1.4(\mathrm{n}=108)$ & 0.022 \\
\hline Preoperative BCVA (LogMAR) & $1.16 \pm 0.03(\mathrm{n}=527)$ & $1.15 \pm 0.06(\mathrm{n}=156)$ & 0.929 \\
\hline Postoperative BCVA (LogMAR) at 1 month & $0.30 \pm 0.01(\mathrm{n}=562)$ & $0.33 \pm 0.02(n=172)$ & 0.201 \\
\hline Postoperative BCVA (LogMAR) at 3 months & $0.18 \pm 0.01(\mathrm{n}=396)$ & $0.23 \pm 0.02(\mathrm{n}=116)$ & 0.002 \\
\hline Postoperative BCVA (LogMAR) at 6 months & $0.14 \pm 0.01(\mathrm{n}=391)$ & $0.13 \pm 0.01(\mathrm{n}=107)$ & 0.551 \\
\hline Postoperative BCVA (LogMAR) at 12 months & $0.11 \pm 0.01(\mathrm{n}=394)$ & $0.11 \pm 0.01(\mathrm{n}=114)$ & 0.540 \\
\hline Preoperative CCT $(\mu \mathrm{m})$ & $661.1 \pm 6.4(\mathrm{n}=365)$ & $666.4 \pm 11.4(\mathrm{n}=117)$ & 0.865 \\
\hline Postoperative CCT at 1 month $(\mu \mathrm{m})$ & $554.9 \pm 9.1(\mathrm{n}=137)$ & $594.2 \pm 16.2(\mathrm{n}=43)$ & 0.036 \\
\hline Postoperative CCT at 3 months $(\mu \mathrm{m})$ & $534.7 \pm 4.8(\mathrm{n}=136)$ & $525.0 \pm 8.3(\mathrm{n}=45)$ & 0.315 \\
\hline Postoperative CCT at 6 months $(\mu \mathrm{m})$ & $538.4 \pm 4.8(\mathrm{n}=180)$ & $516.7 \pm 8.9(\mathrm{n}=53)$ & 0.034 \\
\hline Postoperative CCT at 12 months $(\mu \mathrm{m})$ & $535.3 \pm 2.9(\mathrm{n}=242)$ & $532.8 \pm 5.0(\mathrm{n}=82)$ & 0.667 \\
\hline Re-bubbling; yes (+) (\%) & $255(40.0)(\mathrm{n}=637)$ & $68(34.5)(\mathrm{n}=197)$ & 0.165 \\
\hline Immune rejection $(+)(\%)$ & $7(1.9)(\mathrm{n}=637)$ & $7(3.55)(\mathrm{n}=197)$ & 0.019 \\
\hline
\end{tabular}

Table 2. Comparison of the clinical course between the organ and cold culture groups. ECD endothelial cell density, $S D$ standard deviation, $B C V A$ best-corrected visual acuity, $\log M A R$ logarithm of the minimal angle of resolution, CCT central corneal thickness.

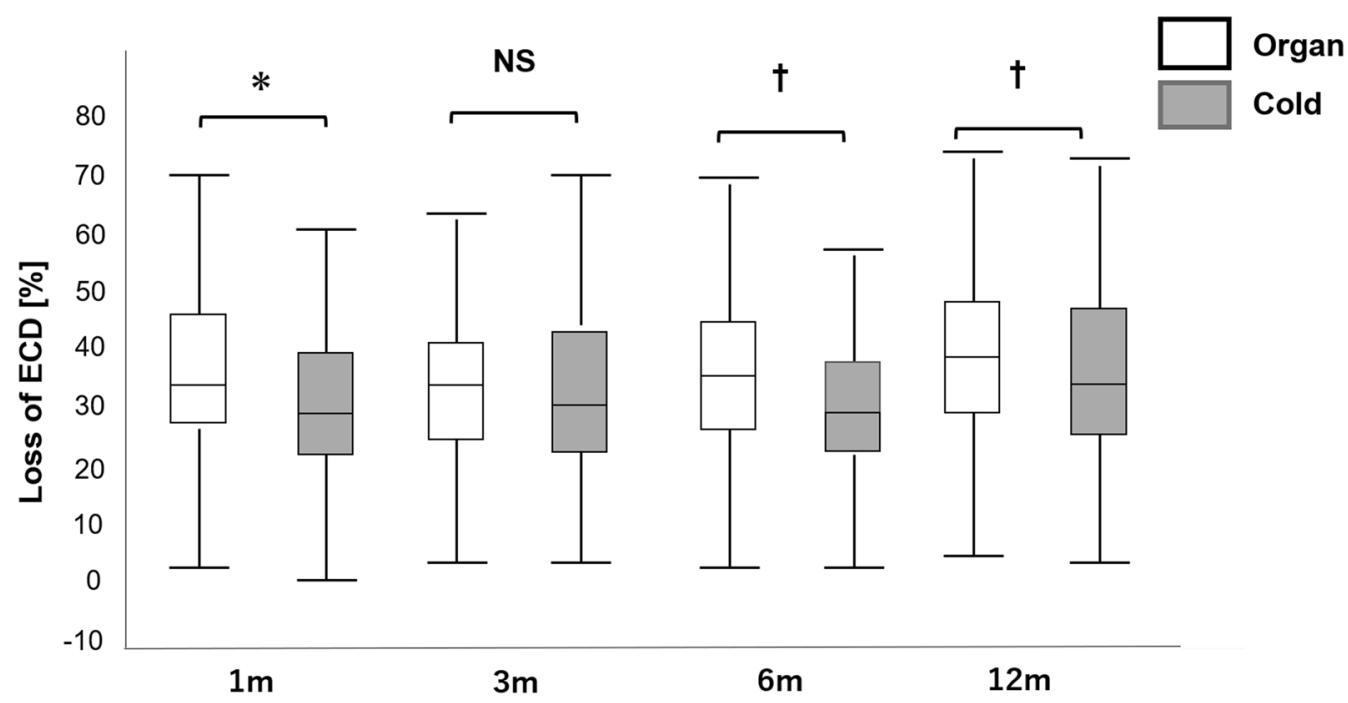

Figure 1. Comparison of ECD loss after DMEK between the organ and cold culture groups. The organ culture group had higher ECD loss rates than the cold culture group $\left({ }^{\star} \mathrm{P}<0.001\right.$ at 1 month, $\mathrm{P}=0.248$ at 3 months, ${ }^{\dagger} \mathrm{P}<0.05[\mathrm{P}=0.003$ at 6 months and $\mathrm{P}=0.022$ at 12 months] after surgery; $\mathrm{t}$-test). Although there was a significant difference between the organ culture and cold culture groups, this difference is clinically irrelevant. The central line means median value, and the whiskers indicates the lowest and highest values. ECD endothelial cell density; DMEK Descemet membrane endothelial keratoplasty, NS not significant.

Correlation. As shown in Table 3 and Supplementary Tables S1 and S2 ECD loss at 1 year postoperatively was strongly associated with the occurrence of re-bubbling in all eyes $(\mathrm{P}<0.001)$ and in those that received triple DMEK $(\mathrm{P}=0.020)$ and DMEK alone $(\mathrm{P}<0.001)$. Finally, re-bubbling was identified as an important risk factor after stepwise variable selection $(\mathrm{P}<0.001)$. 


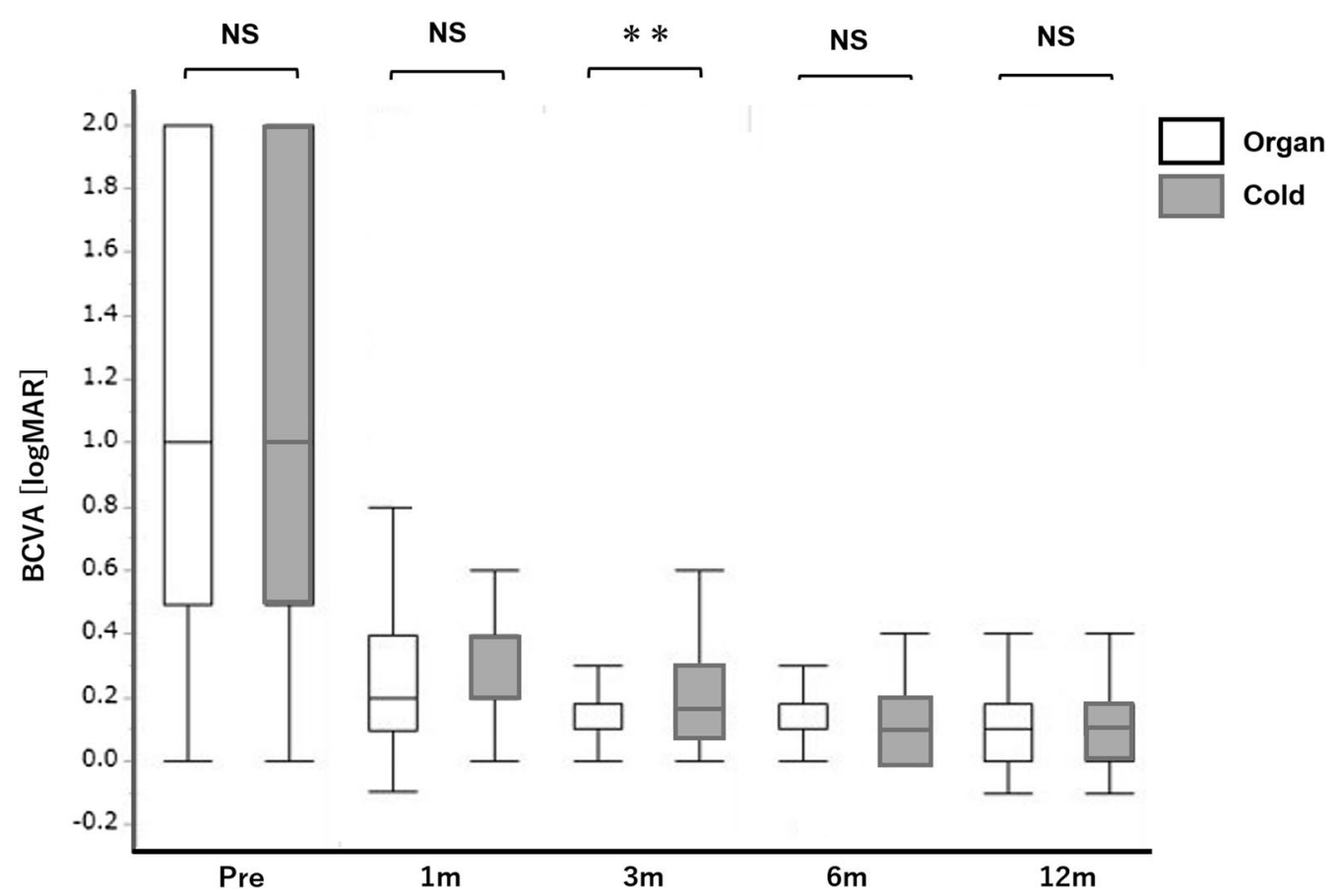

Figure 2. Comparison of the BCVA after DMEK between the organ and cold culture groups. There was a gradual improvement in the BCVA from baseline to 1, 3, 6, and 12 months after surgery. A significant difference was seen between the organ culture and cold culture groups only at 3 months after surgery $\left({ }^{\star \star} \mathrm{P}=0.002\right.$; $\mathrm{t}$-test). The central line means median value, and the whiskers indicates the lowest and highest values. BCVA bestcorrected visual acuity, DMEK Descemet membrane endothelial keratoplasty.

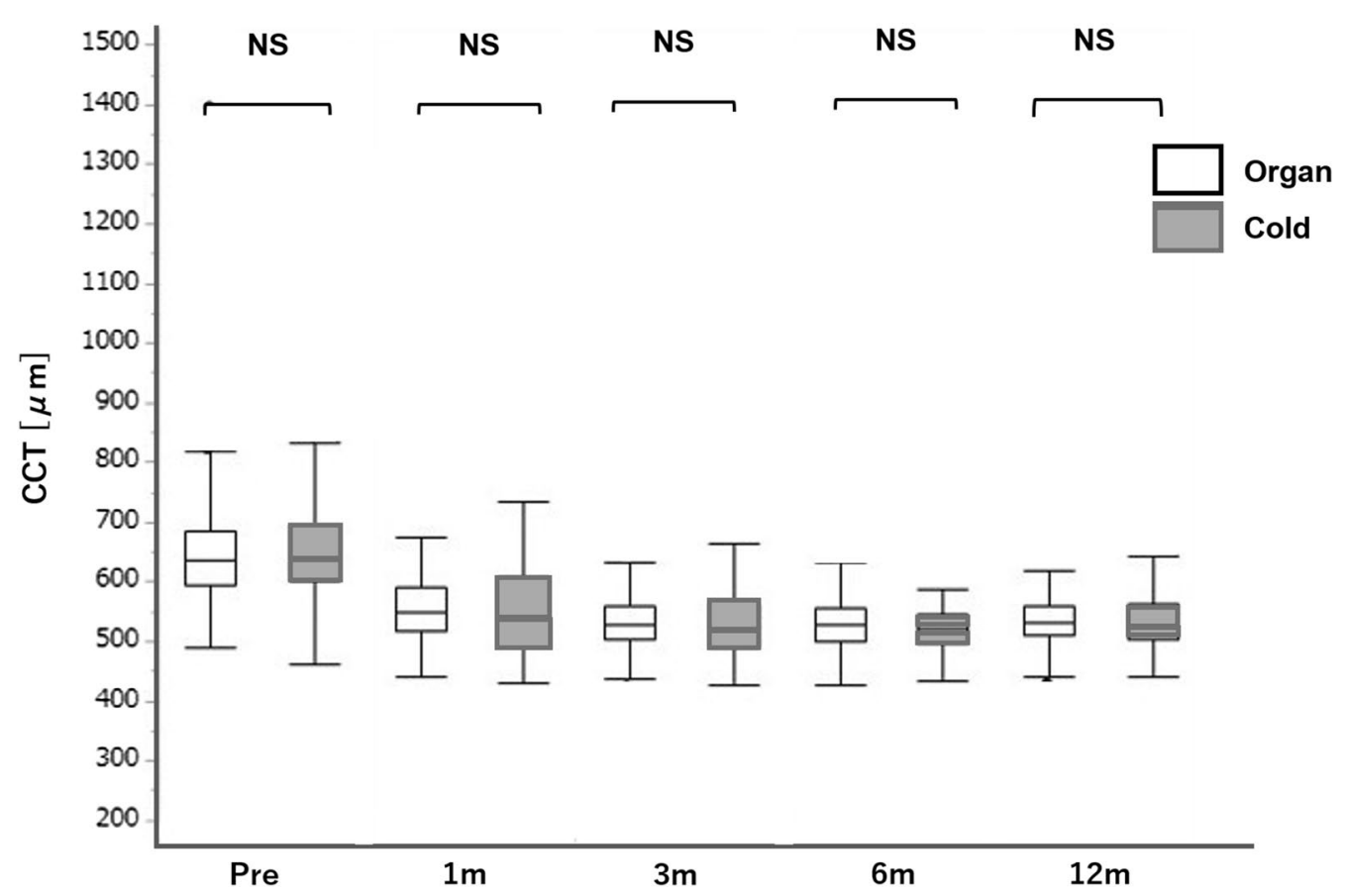

Figure 3. Comparison of the CCT after DMEK between the organ and cold culture groups. There was no significant difference between the organ culture and cold culture groups preoperatively and at 3 and 12 months postoperatively $(\mathrm{P}=0.865,0.315$, and 0.667 ; $\mathrm{t}$-test $)$. The central line means median value, and the whiskers indicates the lowest and highest values. CCT central corneal thickness, DMEK Descemet membrane endothelial keratoplasty. 


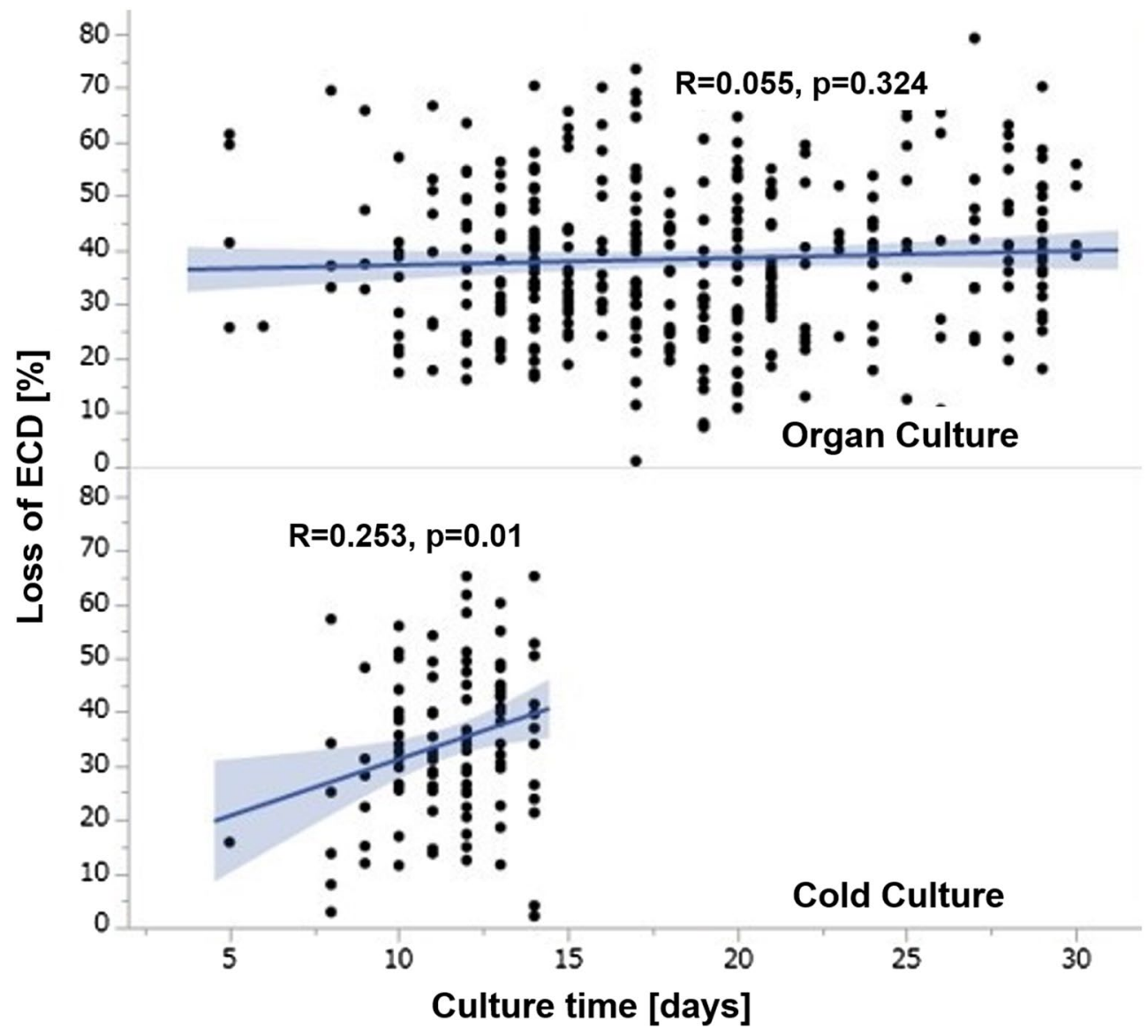

Figure 4. Correlation between the culture time and ECD decline in the organ and cold culture groups. There was no significant correlation between the culture time and ECD decline in the organ culture group $(\mathrm{R}=0.0557$, $\mathrm{P}=0.324)$. The longer culture time had a negative impact on ECD decline in the cold culture group $(\mathrm{R}=0.253$, $\mathrm{P}=0.01)$. ECD endothelial cell density.

\begin{tabular}{|l|c|c|}
\hline Evaluation factors & F ratio & P-value \\
\hline Patient sex (female or male) & 0.149 & 0.699 \\
\hline Patient age & 3.849 & 0.050 \\
\hline Type of tamponade gas (air or 20\% SF6 gas) & 0.795 & 0.373 \\
\hline Type of surgery (triple DMEK or DMEK alone) & 0.143 & 0.705 \\
\hline Re-bubbling (yes) & 16.822 & $<0.001$ \\
\hline Immune rejection (yes) & 1.106 & 0.294 \\
\hline Donor source (organ culture or cold culture) & 2.908 & 0.889 \\
\hline Donor sex (female or male) & 0.295 & 0.587 \\
\hline Donor age & 3.009 & 0.084 \\
\hline
\end{tabular}

Table 3. Factors associated with endothelial cell loss at 1 year after DMEK. SF6, sulphur hexafluoride, DMEK Descemet membrane endothelial keratoplasty, triple DMEK DMEK combined with cataract surgery and intraocular lens implantation, DMEK alone DMEK for pseudophakic eyes.

\section{Discussion}

This retrospective study compared the clinical outcomes between organ culture and cold culture and evaluated the factors associated with ECD loss after DMEK. In general, reasonably good results were obtained at 1 year after DMEK in terms of ECD $\left(1704 \pm 399 \mathrm{cells} / \mathrm{mm}^{2}\right)$ and ECD loss $(37.0 \pm 14.3 \%)$.

Some results from our comparison between organ culture and cold culture are not consistent with those from previous studies. Although the re-bubbling rates were higher in cold culture in previous studies ${ }^{16-18}$, there was no difference found in our study. Despite similar results for the BCVA and CCT, ECD loss seemed to be greater 
in the organ culture at 1,6 , and 12 months postoperatively. The difference in the values could be attributed to this divergence.

This study allows for the following conclusions to be drawn.

(1) Immune reactions are more common in cold cultures.

(2) Although organ culture yields higher ECD loss rates than does cold culture, the difference is clinically

irrelevant.

(3) There is a strong correlation between the occurrence of re-bubbling and ECD loss at 1 year after DMEK.

Interestingly, the rate of immune rejection was significantly higher in the cold culture than in the organ culture $(\mathrm{P}=0.009$, Pearson's chi-squared test). Some reports suggest the possibility that a longer storage time could decrease the immunogenicity of the donor and therefore decrease graft rejection ${ }^{19,20}$. A possible explanation for this could be that the outflow of bone marrow-derived antigen-presenting cells from the donor tissue during storage could suppress graft rejection. In fact, the storage time was longer in the organ culture than in the cold culture in our study. However, it is uncertain whether a longer storage time could reduce graft rejection in DMEK, in which only a monolayer of endothelium without donor bone marrow-derived antigen-presenting cells is transplanted.

A possible explanation for the greater ECD loss in the organ culture could be the longer storage time. In fact, the mean storage time in the organ culture $(18.7 \pm 0.2$ days) was significantly longer than that in the cold culture (11.4 \pm 0.4 days). However, this hypothesis was denied because there was no correlation found between the storage time and ECD loss in the organ culture, as shown in Fig. 4. Although there was no significant correlation between the culture time and ECD loss in the organ culture, the longer culture time had a negative impact on ECD loss in the cold culture. It is possible that the evaluation of the preoperative ECD is inaccurate. The lack of evaluation of ECD immediately before surgery could also be a limitation of this study because it has been reported that many dead cells can be detected after a long storage time ${ }^{21-24}$.

In our study, we performed a multivariable regression analysis to identify the risk factors for ECD loss. We found a strong relationship between re-bubbling and ECD loss. There was no significant difference in the occurrence of re-bubbling between the two groups (organ culture and cold culture). However, it remains uncertain whether re-bubbling procedures itself cause ECD loss or whether endothelial cells with low pump function (low viability or dead cells) cause graft detachment necessitating rebubbling.

Our multivariable regression analysis included some clinically important factors, such as the type of tamponade (air or 20\% SF6 gas), the type of surgery (triple DMEK or DMEK alone), re-bubbling, immune rejection, and age and sex. Although the use of SF6 gas significantly reduced the rates of graft detachment and re-bubbling ${ }^{15,21}$, there was no significant difference in the ECD loss between SF6 and air. Although Busin et al. reported that triple DMEK could be a risk factor for re-bubbling ${ }^{10}$, the type of surgery was not found as a risk factor in our study.

The strengths of this study are the retrospective analysis within a prospective trial database for a single disease (FECD) in a large sample, determination of surgical outcomes by two experienced DMEK surgeons (both surgeons have experiences of $>4,000$ DMEKs ${ }^{25}$, and consideration of clinically important factors. In contrast, the limitations of this study include its retrospective nature, lack of precise donor information (death to harvest time), and missing values. Another limitation is the lack of precise evaluation of the graft/descemetorhexis size and actual times of re-bubbling. Feng et al. suggested that additional re-bubbling (more than twice) could have a negative impact on ECD loss ${ }^{26}$.

In conclusion, re-bubbling procedures or the innate graft viability that requires re-bubbling might have a negative impact on the final ECD outcome or ECD loss. Surgeons should reconsider the impact or the necessity of re-bubbling in such cases. Furthermore, both organ- and cold-donor culture media can be used safely in patients, as they have similar benefits.

\section{Methods}

Patient selection. Patients with FECD who underwent DMEK were selected from the Cologne DMEK database using Research Electronic Data Capture, which is a secure, website-based application designed to support data capture for research studies. The data of consecutive patients who underwent DMEK from 2011 to 2016 were obtained prospectively from medical records and reviewed via a retrospective analysis. The study was performed in accordance with the tenets of the Declaration of Helsinki; all study participants provided their informed consent; and the protocol was approved by the Ethics Commission of the Medical Faculty of the University of Cologne (number: 14-373). Although the FECD cases included those that received triple DMEK, DMEK alone, and phakic DMEK, those who underwent phakic DMEK were excluded because of their characteristics, such as younger age and a shallow anterior chamber (AC). Patients with pre-existing diseases/conditions that would influence the final visual acuity were also excluded (macular degeneration, wet-type AMD, amblyopia, history of trauma, uncontrolled glaucoma with advanced visual field loss, pre-existing AC lens, or history of glaucoma surgery, or vitrectomy). As for the patients with bilateral involvement, only the first eye was included. Cases of primary graft failure were also excluded from this study.

Surgical technique and postoperative medication. As in our previous reports, standard DMEK procedures were performed by two experienced surgeons $(\mathrm{CC} \text { and } \mathrm{BB})^{13,25}$. Corneal donors were obtained from eye banks from Germany or the USA. In brief, the entire cornea was fixed with a suction block (Moria SA, Antomy, France) and stained with $0.06 \%$ trypan blue (Vision Blue, D.O.R.C. International, Zuidland, the Netherlands) for 5 to $10 \mathrm{~s}$. The DM was peeled off and separated from the stroma. For proper orientation, asymmetric marking was used ${ }^{27}$ and punched in the proper diameter $(8.0$ to $10.0 \mathrm{~mm})$. A DMEK graft was set in the intra-ocular lens (IOL) cartridge (AT Smart Cartridge, Carl Zeiss Meditec, Jena, Germany) and afterwards in the IOL injector 
(AT Shooter A2-2000, Zeiss Meditec) ${ }^{28}$. Under general anaesthesia, partial iridectomy was enlarged using Vannas capsulotomy scissors (Asico, Westmont, IL, USA), and descemetorhexis was performed under air within a diameter larger than the graft size using a Price hook ${ }^{28}$. The DMEK graft was placed into the AC, unfolded, and fixed with air or $20 \%$ SF6 gas (Alcon Laboratories, Fort Worth, TX, USA). In the triple DMEK cases, phacoemulsification and IOL implantation were performed before DMEK. Until air or gas disappeared, all the patients were instructed to assume the supine position under continuous monitoring of intra-ocular pressure. Pilocarpine $2 \%$ eye drops (Bausch \& Lomb) were used three times a day. Postoperative medications included topical antibiotics (ofloxacin) (Floxal EDO; Mann Pharma, Berlin, Germany) administered four times daily for 2 weeks and prednisolone acetate 1\% (Inflanefran Forte; Pharm-Allergan, Ettlingen, Germany) administered five times daily for the first postoperative month; steroids were then tapered once per month and administered for 24 months. (Briefly, patients received 5 drops per day in the first postoperative month, 4 drops per day in the second month postoperatively, 3 drops per day in the third month postoperatively, 2 drops per day in the fourth postoperative month and one drop per day from the fifth month postoperatively until two years after surgery.) Lubricant eye drops (Hylo Care; Ursapharm, Saarbrücken, Germany) were used for 5 days. The indication for re-bubbling was determined using an anterior segment OCT device (AS-OCT) within 14 days after surgery. In cases where the graft detachment reached the pupil area, the re-bubbling procedure was performed under the operating microscope in the operating room.

Clinical data. The following donor information was included in the database: age (years), sex (female/male), ECD (cells $/ \mathrm{mm}^{2}$ ), source of tissue, and storage time (days). The donor sources were divided into two groups: organ culture (stored in a Dulbecco modified Eagle's medium containing streptomycin and penicillin [Biochrom, Berlin, Germany] as well as faetal calf serum [Linaris Bettingen am Main, Germany] at a warm temperature $\left[37^{\circ} \mathrm{C}\right]$ ) and cold culture (stored in Optisol-GS [Bauch \& Lomb, Irvine, CA, USA] at a cold temperature [ $\left.5{ }^{\circ} \mathrm{C}\right]$ ). In the organ culture group, the culture medium that consisted of Dulbecco modified Eagle's medium with faetal calf serum was supplemented with dextran (10\% dextran T500) medium before transplantation (average interval, $24-48 \mathrm{~h}$ ). The storage time in both groups was defined from the timepoint when corneoscleral buttons were placed into the culture medium to the day of surgery.

After surgery, all patients attended follow-up visits as per standard protocols. In addition to the usual ophthalmic examination using slit-lamp microscopy and funduscopy of the retina, the following parameters were documented: BCVA (measured as decimal visual acuity and converted to logMAR units for statistical analysis), ECD [measured via specular microscopy of the corneal endothelium (EM-3000, Tomey, Nagoya, Japan)], CCT [measured via corneal tomography (Pentacam, Oculus, Wetzlar, Germany)], graft attachment [evaluated using the AS-OCT (Heidelberg Engineering GmbH, Germany)], and posterior segment values [evaluated using a macular SD-OCT (Spectralis HRApOCT; Heidelberg Engineering GmbH)] before and 1, 3, 6, and 12 months after DMEK. A macular OCT was used for exclusion of the existence of macular degeneration or wet-type AMD. The preoperative (baseline) ECD was evaluated before storage.

Evaluation factors and statistics. The clinical factors used for the analysis are numbered (1) to (9) below.

The patient-related factors were as follows: (1) sex and (2) age.

The procedure- and clinical course-related factors were as follows: (3) type of tamponade (air or 20\% SF6 gas), (4) type of surgery (triple DMEK or DMEK alone), (5) re-bubbling (yes/no), and (6) immune rejection (yes). The donor-related factors were as follows: (7) sex, (8) age, and (9) source.

Statistical analyses. Statistical analysis was performed using the JMP Pro software version 14.0.0 (SAS Institute, Cary, NC, USA). To compare the continuous variables in each group, we used the t-test; to compare the nominal variables, we applied Pearson's chi-squared test. Pearson correlation analysis was used for the univariable analyses. Associations between the factors and ECD loss rates at 12 months were examined using multivariable regression analysis and second-order polynomial regression after stepwise variable selection (using the minimum Bayesian information criterion and increasing the number of variables). To exclude potential multicollinearity factors between the variables, we checked the variance inflation factor. The statistical significance level was set at $\mathrm{P}$-values of $<0.05$.

Received: 30 January 2020; Accepted: 10 June 2020

Published online: 06 July 2020

\section{References}

1. Melles, G. R., Ong, T. S., Ververs, B. \& van der Wees, J. Descemet membrane endothelial keratoplasty (DMEK). Cornea 25, 987-990 (2006).

2. Guerra, F. P., Anshu, A., Price, M. O., Giebel, A. W. \& Price, F. W. Descemet's membrane endothelial keratoplasty: prospective study of 1-year visual outcomes, graft survival, and endothelial cell loss. Ophthalmology 118, 2368-2373 (2011).

3. Terry, M. A. et al. Standardized DMEK technique: reducing complications using Prestripped tissue, novel glass injector, and sulfur hexafluoride (SF6) gas. Cornea 34, 845-852 (2015).

4. Schlögl, A., Tourtas, T., Kruse, F. E. \& Weller, J. M. Long-term clinical outcome after descemet membrane endothelial keratoplasty. Am. J. Ophthalmol. 169, 218-226 (2016).

5. Flockerzi, E. et al. Trends in corneal transplantation from 2001 to 2016 in Germany: a report of the DOG-section cornea and its Keratoplasty Registry. Am. J. Ophthalmol. 188, 91-98 (2018).

6. Dapena, I., Ham, L., Netuková, M., van der Wees, J. \& Melles, G. R. Incidence of early allograft rejection after descemet membrane endothelial keratoplasty. Cornea 30, 1341-1345 (2011). 
7. Anshu, A., Price, M. O. \& Price, F. W. Jr. Risk of corneal transplant rejection significantly reduced with descemet's membrane endothelial keratoplasty. Ophthalmology 119, 536-540 (2012).

8. Hos, D. et al. Incidence and clinical course of immune reactions after descemet membrane endothelial keratoplasty: retrospective analysis of 1000 consecutive eyes. Ophthalmology 124, 512-518 (2017).

9. Hos, D. et al. Immune reactions after modern lamellar (DALK, DSAEK, DMEK) versus conventional penetrating corneal transplantation. Prog. Retin. Eye Res. 73, 100768 (2019).

10. Busin, M. et al. Clinical outcomes of preloaded descemet membrane endothelial keratoplasty grafts with endothelium tri-folded inwards. Am. J. Ophthalmol. 193, 106-113 (2018).

11. Ang, M. et al. Descemet membrane endothelial keratoplasty: preliminary results of a donor insertion pull-through technique using a donor mat device. Am. J. Ophthalmol. 171, 27-34 (2016).

12. Ham, L. et al. Midterm results of descemet membrane endothelial keratoplasty: 4 to 7 years clinical outcome. Am. J. Ophthalmol. 171, 113-121 (2016).

13. Schrittenlocher, S., Bachmann, B. \& Cursiefen, C. Impact of donor tissue diameter on postoperative central endothelial cell density in Descemet membrane endothelial keratoplasty. Acta Ophthalmol. 97, e618-e622 (2019).

14. Terry, M. A., Shamie, N., Straiko, M. D., Friend, D. J. \& Davis-Boozer, D. Endothelial keratoplasty: the relationship between donor tissue storage time and donor endothelial survival. Ophthalmology 118, 36-40 (2011).

15. Schaub, F. et al. One-year outcome after descemet membrane endothelial keratoplasty (DMEK) comparing sulfur hexafluoride (SF6) $20 \%$ versus $100 \%$ air for anterior chamber tamponade. Br. J. Ophthalmol. 101, 902-908 (2017).

16. Salla, S., Kruse, F. E., Walter, P. \& Menzel-Severing, J. Supplementation of organ culture medium with dextran is not required in pre-stripped human donor tissue for DMEK surgery. Cell Tissue Bank 20, 193-200 (2019).

17. Abdin, A. et al. Negative impact of dextran in organ culture media for pre-stripped tissue preservation on DMEK (Descemet membrane endothelial keratoplasty) outcome. Graefes Arch. Clin. Exp. Ophthalmol. 256, 2135-2142 (2018).

18. Laaser, K. et al. Donor tissue culture conditions and outcome after Descemet membrane endothelial keratoplasty. Am. J. Ophthalmol. 151, 1007-1018.e2 (2011).

19. Zhang, X. et al. Depletion of passenger leukocytes from corneal grafts: an effective means of promoting transplant survival?. Invest. Ophthalmol. Vis. Sci. 50, 3137-3144 (2009).

20. Kamiya, K. et al. Preservation of donor cornea prevents corneal allograft rejection by inhibiting induction of alloimmunity. Exp. Eye Res. 70, 737-743 (2000).

21. Güell, J. L., Morral, M., Gris, O., Elies, D. \& Manero, F. Comparison of sulfur hexafluoride 20\% versus air tamponade in Descemet membrane endothelial keratoplasty. Ophthalmology 122, 1757-1764 (2015).

22. Schrittenlocher, S. et al. Evolution of consecutive Descemet membrane endothelial keratoplasty outcomes throughout a 5-year period performed by two experienced surgeons. Am. J. Ophthalmol. 190, 171-178 (2018).

23. Kitazawa, K. et al. The existence of dead cells in donor corneal endothelium preserved with storage media. Br. J. Ophthalmol. 101, 1725-1730 (2017).

24. Rodríguez-Calvo de Mora, M. et al. Association between graft storage time and donor age with endothelial cell density and graft adherence after descemet membrane endothelial keratoplasty. JAMA Ophthalmol. 134, 91-94 (2016).

25. Stulting, R. D. et al. Cornea Preservation Time Study Group. Factors associated with graft rejection in the cornea preservation time study. Am. J. Ophthalmol. 196, 197-207 (2018).

26. Feng, M. T., Price, M. O., Miller, J. M. \& Price, F. W. Jr. Air reinjection and endothelial cell density in Descemet membrane endothelial keratoplasty: five-year follow-up. J. Cataract Refract. Surg. 40, 1116-1121 (2014).

27. Bachmann, B. O., Laaser, K., Cursiefen, C. \& Kruse, F. E. A method to confirm correct orientation of descemet membrane during descemet membrane endothelial keratoplasty. Am. J. Ophthalmol. 149, 922-925.e2 (2010).

28. Kruse, F. E. et al. A stepwise approach to donor preparation and insertion increases safety and outcome of descemet membrane endothelial keratoplasty. Cornea 30, 580-587 (2011).

\section{Acknowledgements}

This work was supported by DFG FOR 2240 (www.for2240.de), EU ARREST and BLINDNESS (www.arrestblin dness.eu), the Japan Eye Bank Association (https://www.j-eyebank.or.jp), and the Alexander von Humboldt Foundation (https://www.humboldt-foundation.de/web/home.html).

\section{Author contributions}

T.H.: writing, reviewing, and editing of the manuscript; S.S.: curation of the data and editing of the manuscript; S.S.: reviewing and editing of the manuscript; V.N.H.L.: curation and analysis of the data and investigation; M.M.: reviewing of the manuscript and investigation; J.F.: statistical analysis of the data and investigation; B.B.: administration of the project and investigation; C.C.: supervision and validation; All authors critically checked the manuscript and approved its submission.

\section{Competing interests}

The authors declare no competing interests.

\section{Additional information}

Supplementary information is available for this paper at https://doi.org/10.1038/s41598-020-68023-0.

Correspondence and requests for materials should be addressed to T.H.

Reprints and permissions information is available at www.nature.com/reprints.

Publisher's note Springer Nature remains neutral with regard to jurisdictional claims in published maps and institutional affiliations. 
(c) (i) Open Access This article is licensed under a Creative Commons Attribution 4.0 International cc) License, which permits use, sharing, adaptation, distribution and reproduction in any medium or format, as long as you give appropriate credit to the original author(s) and the source, provide a link to the Creative Commons license, and indicate if changes were made. The images or other third party material in this article are included in the article's Creative Commons license, unless indicated otherwise in a credit line to the material. If material is not included in the article's Creative Commons license and your intended use is not permitted by statutory regulation or exceeds the permitted use, you will need to obtain permission directly from the copyright holder. To view a copy of this license, visit http://creativecommons.org/licenses/by/4.0/.

(C) The Author(s) 2020 